\title{
First Passage Time Analysis on Climate Indices
}

\author{
Peter C. Chu \\ Naval Ocean Analysis and Prediction Laboratory, Naval Postgraduate School, Monterey, California
}

(Manuscript received 12 February 2007, in final form 11 June 2007)

\begin{abstract}
Climate variability is simply represented by teleconnection patterns such as the Arctic Oscillation (AO), Antarctic Oscillation (AAO), North Atlantic Oscillation (NAO), Pacific-North American pattern (PNA), and Southern Oscillation (SO) with associated indices. Two approaches can be used to predict the indices: forward and backward methods. The forward method is commonly used to predict the index fluctuation $\rho$ at time $t$ with a given temporal increment $\tau$. Using this method, it was found that the index (such as for NAO) has the Brownian fluctuations. On the basis of the first passage time (FPT) concept, the backward method is introduced in this study to predict the typical time span $(\tau)$ needed to generate a fluctuation in the index of a given increment $\rho$. After the five monthly indices (AO, AAO, NAO, PNA, and SO) run through the past history, the FPT density functions are obtained. FPT presents a new way to detect the temporal variability of the climate indices. The basic features for the index prediction are also discussed.
\end{abstract}

\section{Introduction}

Complexity in climate systems makes prediction difficult. One way to simplify the climate systems is to represent low-frequency variability of atmospheric circulations by teleconnection patterns, such as the Arctic Oscillation (AO), Antarctic Oscillation (AAO), North Atlantic Oscillation (NAO), Pacific-North American pattern (PNA), and Southern Oscillation (SO). Temporally varying indices, $s(t)$, were calculated for these patterns, where $t$ denotes time.

Among them, the SO index (SOI) was first to utilize equivalent barotropic seesaw in atmospheric pressure between the southeastern tropical Pacific and the Australian-Indonesian regions (Walker and Bliss 1937). A popular formula for calculating the monthly SOI is proposed by the Australian Bureau of Meteorology:

$$
s(t)=10 \times \frac{p_{\text {diff }}(t)-\left\langle p_{\text {diff }}\right\rangle}{\operatorname{SD}\left(p_{\text {diff }}\right)} .
$$

Here, $p_{\text {diff }}$ is the mean sea level pressure of Tahiti minus that of Dawin for that month; $\left\langle p_{\text {diff }}\right\rangle$ is the long-term average of $p_{\text {diff }}$ for the month in question; and $\operatorname{SD}\left(p_{\text {diff }}\right)$

Corresponding author address: Peter C. Chu, Naval Ocean Analysis and Prediction Laboratory, Naval Postgraduate School, Monterey, CA 93943.

E-mail: pcchu@nps.edu is the long-term standard deviation of $p_{\text {diff }}$ for the month in question. The SOI ranges from about -35 to about +35 .

The indices of the other oscillations (AO, AAO, NAO, PNA) are the expansions of similar seesaw phenomena such as the Northern Hemisphere annular mode (sometimes called AO) and the two patterns (i.e., NAO and AO) as "two paradigms of the same phenomenon" (Wallace 2000). These oscillation patterns are usually calculated on the basis of the Rotated Principal Component Analysis (RPCA; Barnston and Livezey 1987). Monthly (AO, AAO, NAO, and PNA) indices are constructed by projecting the monthly mean $(1000,700,500$, and $500 \mathrm{hPa})$ height anomalies onto the leading EOF mode. The time series are normalized by the standard deviation of the monthly index. Since the loading pattern of $\mathrm{AO}, \mathrm{AAO}, \mathrm{NAO}$, and PNA is obtained using the monthly mean height anomaly dataset, the index corresponding to each loading pattern becomes one when it is normalized by the standard deviation of the monthly index. (Detailed information is available online at http://www.cpc.noaa.gov/.)

Long-term time series of the climate indices are invaluable sources of information to represent the complex seasonal, interannual, and interdecadal variabilities of the climate systems. For example, sustained negative values of the SOI often indicate El Niño episodes. These negative values are usually accompanied by sustained warming of the central and eastern tropi- 
cal Pacific Ocean, a decrease in the strength of the Pacific trade winds, and a reduction in rainfall over eastern and northern Australia. Positive values of the SOI are associated with stronger Pacific trade winds and warmer sea temperatures to the north of Australia, popularly known as a La Niña episode. Waters in the central and eastern tropical Pacific Ocean become cooler during this time. Together these give an increased probability that eastern and northern Australia will be wetter than normal. The SOI has been used in numerous studies as an indicator of the status of the ENSO phenomenon and as a predictor for global and regional climatic prediction such as temperature (e.g., Smith and Sterns 1993), precipitation (e.g., Ropelewski and Halpert 1987), and agricultural products (e.g., Rimmington and Nicholls 1993). The other indices have similar features.

How to effectively predict the climate indices has practical significance because of their connection to the large-scale atmospheric circulations. Usually, these indices are treated as time series and statistical predictions are conducted (forward method). For example, the singular spectrum analysis (Keppenne and Ghil 1992), the wavelet analysis (Torrence and Campo 1998), and the nonlinear analog analysis (Drosdowsky 1994) were used to obtain the dominant frequencies of the SOI time series. The power-law correlations were found for the self-affine properties of the SOI (Ausloos and Ivanova 2001).

It may be possible to use an alternative "backward" method that predicts a typical time span $(\tau)$ needed to generate a fluctuation in the index of a given increment $\rho$. This method is established on the basis of the first passage time (FPT) concept, which is widely used in many disciplines, such as physics, chemistry, biology, and economics, but not in meteorology until recently when Chu et al. (2002a,b) used FPT to study oceanatmospheric model predictability. The major purpose of this paper is to show the usefulness of FPT on the climate index prediction.

The rest of the paper is outlined as follows. Section 2 discusses the two approaches to predict the climate indices. Section 3 shows the FPT concept applied in this paper. Section 4 investigates the sensitivity of the FPT density function to the index reduction $\rho$. Section 5 shows the power-law dependence of optimal FPT on the index reduction density $\rho$ and classifies the variability of the climate indices as the Brownian motion using the FPT. Finally, section 6 presents the conclusions.

\section{Forward and backward approaches of prediction}

Monthly varying climate indices from the National Oceanic and Atmospheric Administration (NOAA)
Climate Prediction Center show randomness (Fig. 1) with poor predictability because their phases and amplitudes are rather unpredictable as both involve many (time and space) scales that are often intrinsic to chaotic behavior [see reviews for the NAO index (Palmer 2000; Greatbatch 2000; Wanner et al. 2001)]. The histograms of the monthly mean indices show Gaussiantype distributions with mean values near zero for all the indices (AO: -0.136; AAO: -0.01; NAO: -0.004 ; PNA: 0.018; SO: -0.077$)$ and with standard deviations near 1 for the AO (0.981), AAO (0.989), and NAO (0.985) indices, and near 10 for the SOI (10.5) (Fig. 2).

Both forward and backward approaches exist in index prediction. The forward approach predicts the change of the index $(\rho)$ at time $t$ with a given temporal increment $\tau$ from analyzing single or multiple time series. Because of stochastic nature, the probability density function (PDF) $p[\rho(t), \tau]$ should be constructed first. Collette and Ausloos (2004) analyzed the NAO monthly index (single time series) from 1825 to 2002 and found that the long-range time correlations are similar to Brownian fluctuations. The distribution functions of the NAO monthly index fluctuations have a form close to a Gaussian for all time lags. This indicates the lack of predictive power of the present NAO monthly index. Lind et al. (2005) used the standard Markov analysis to get the Chapman-Kolmogorov equation for the conditional PDF of the increments $\rho$ of the NAO index over different time intervals $\tau$ and to compute the diffusion and drift coefficients $\left(D^{(1)}, D^{(2)}\right)$ from the first two moments of such probability distribution. The random variable $\rho(t)$ is found to satisfy the Langevin equation:

$$
\frac{d \rho(t)}{d t}=D^{(1)}[\rho(t), t]+\eta(t) \sqrt{D^{(2)}[\rho(t), t]},
$$

where $\eta(t)$ is a fluctuating $\delta$-correlated force with Gaussian statistics, such that

$$
\left\langle\eta(t) \eta\left(t^{\prime}\right)\right\rangle=2 \delta\left(t-t^{\prime}\right)
$$

Maharaj and Wheeler (2005) predict the daily bivariate index (multiple time series) of the Madden-Julian oscillation using seasonally varying vector autoregressive (VAR) models. The first-order VAR model on the original (nondifferenced) time series was found to be the most satisfactory for forecasting the index beyond a few days. Although this model shows no strong skill advantage over a lagged regression technique, it has the convenience of employing only a single set of equations to make predictions for multiple forecast horizons.

The backward approach predicts the typical time $\operatorname{span}(\tau)$ needed to generate a fluctuation in the index of 
(a)

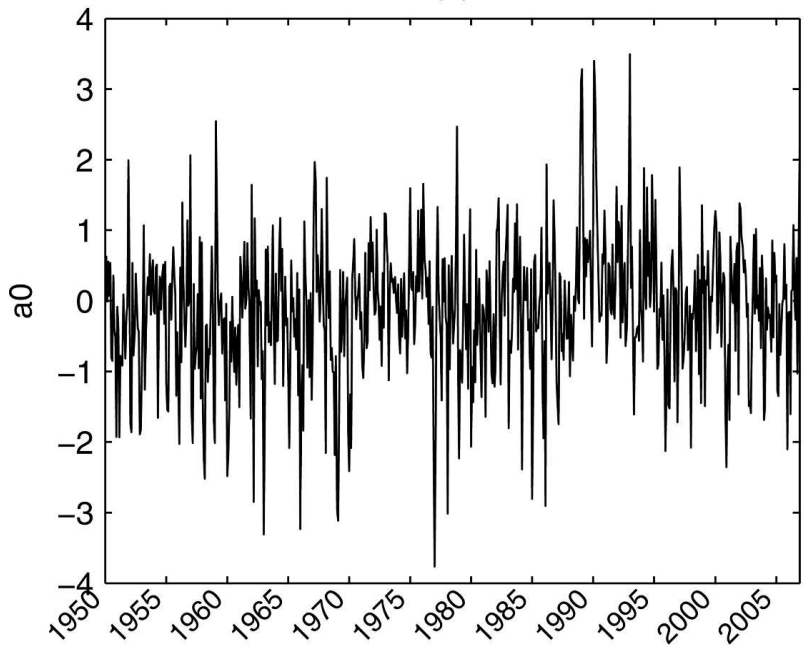

(c)

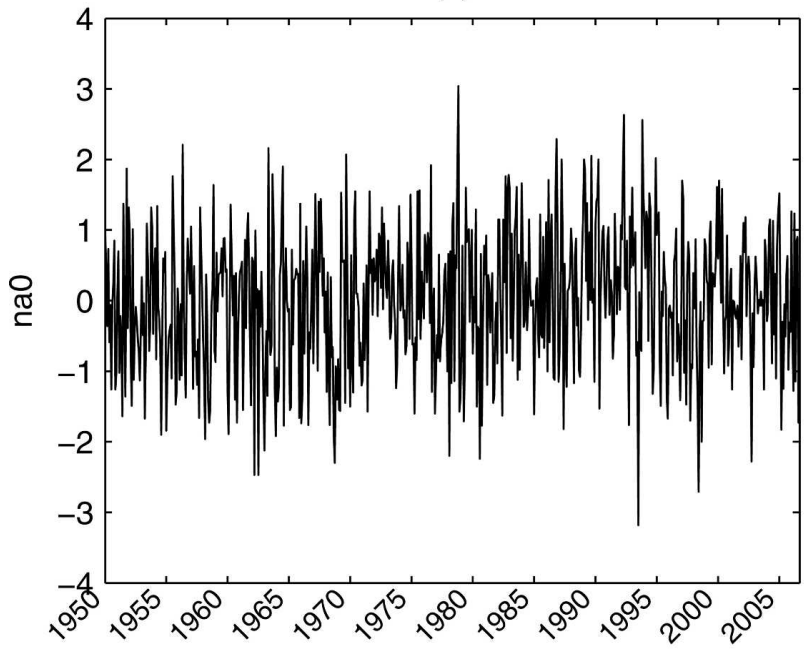

(b)

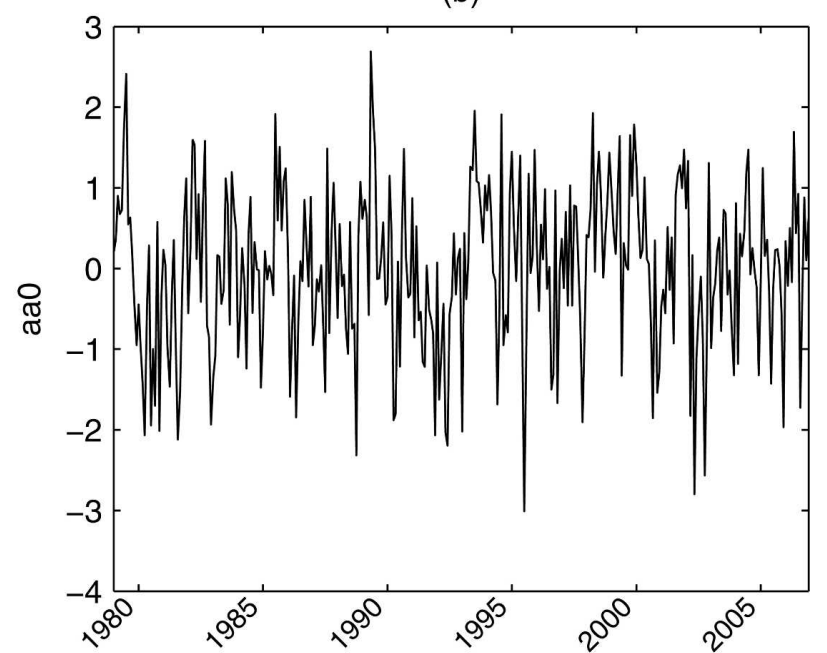

(d)

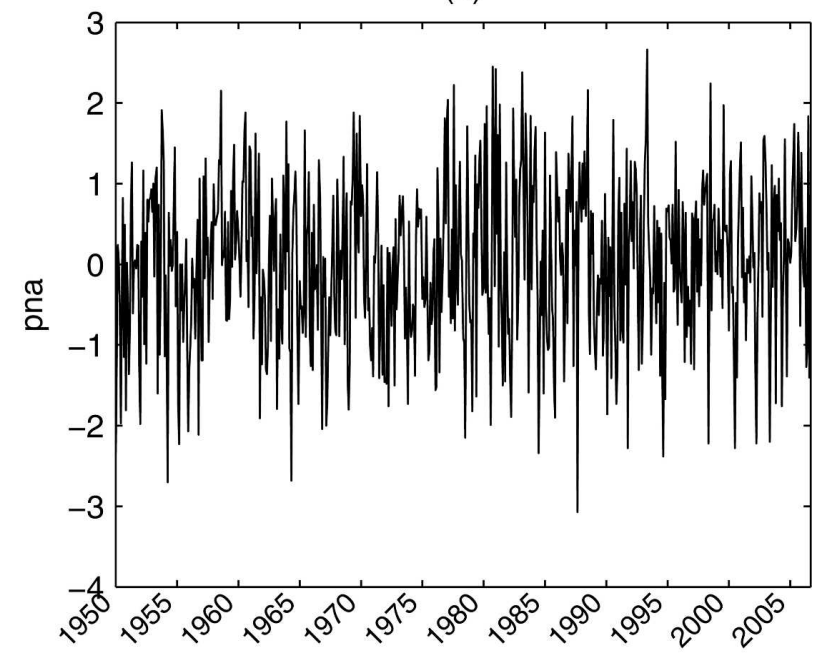

(e)

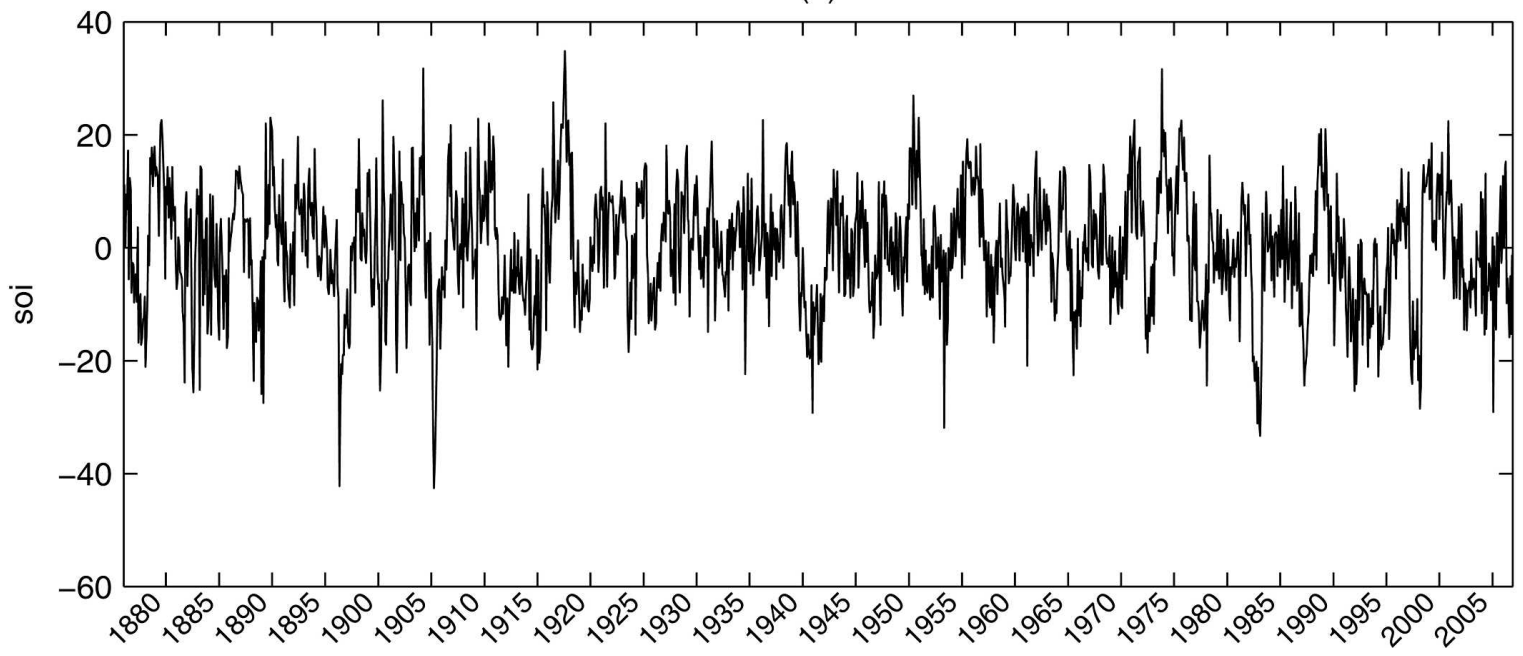

FIG. 1. The monthly mean climate index data obtained from NOAA Climate Prediction Center for the (a) AO, (b) AAO, (c) NAO, (d) PNA, and (e) SO. (Detailed information is available online at http://www.cpc.noaa.gov/.) 
(a)

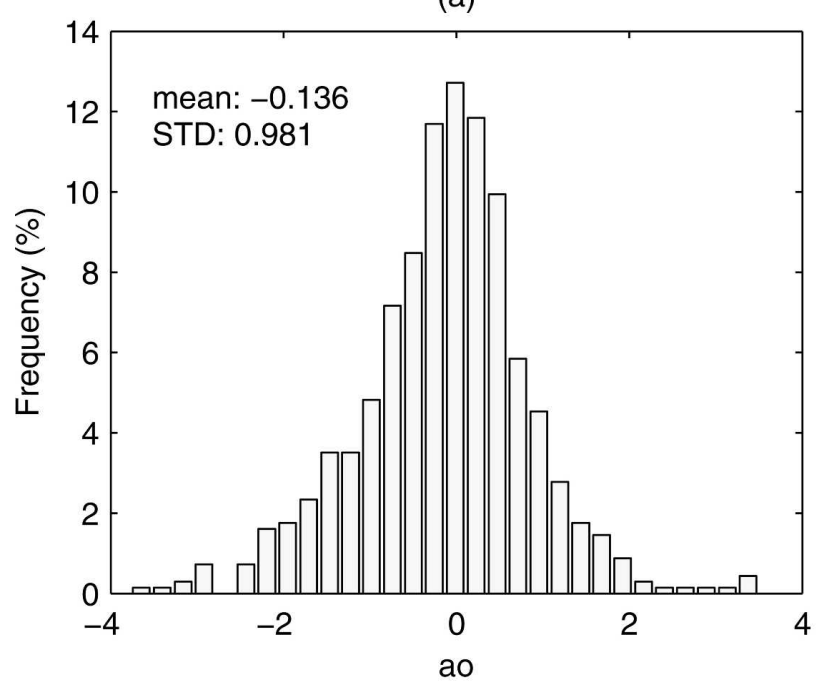

(c)

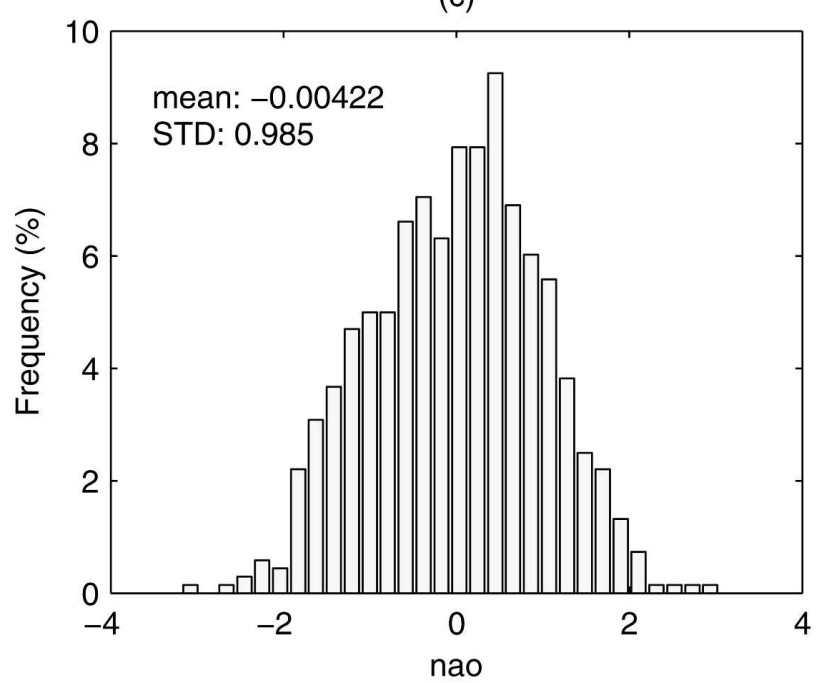

(b)

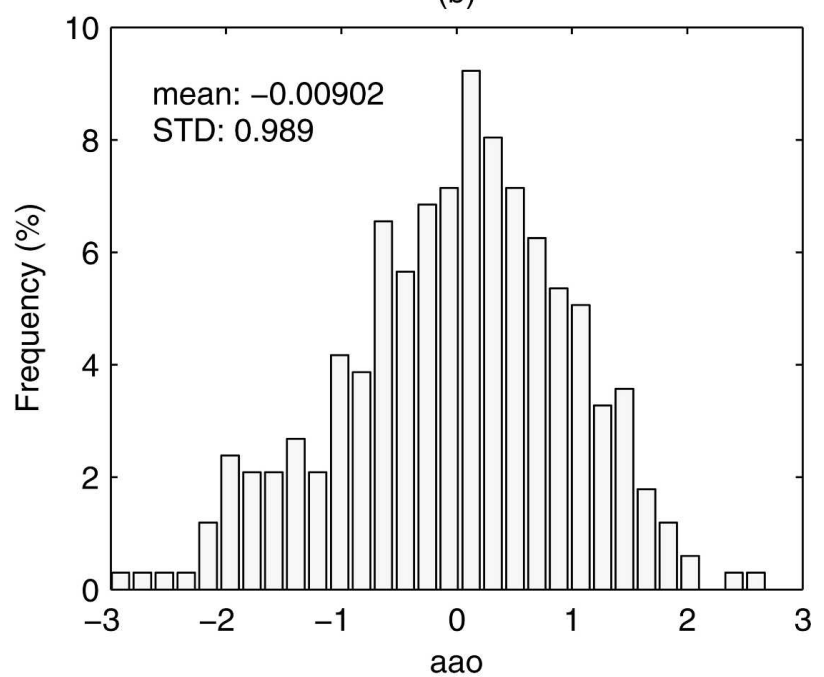

(d)

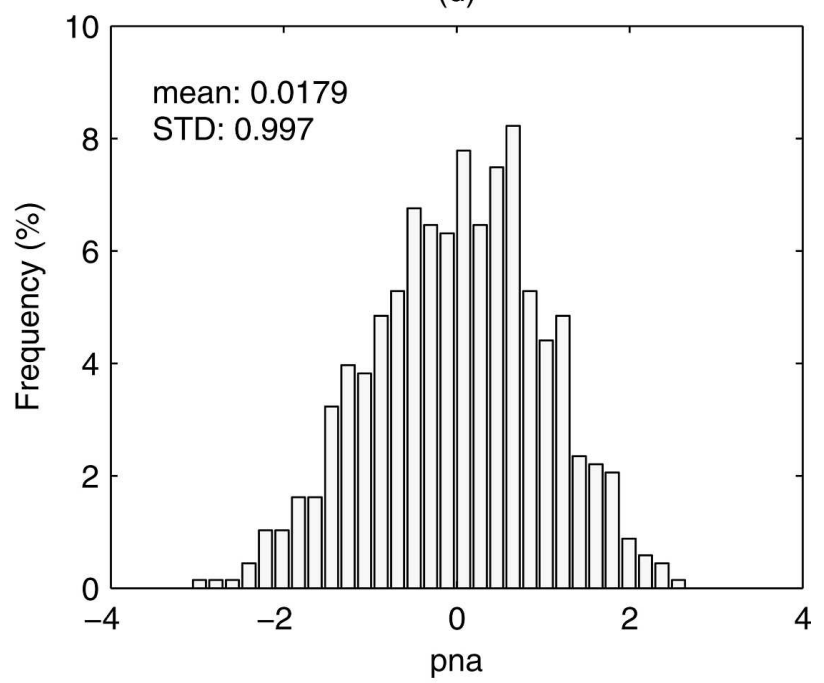

(e)

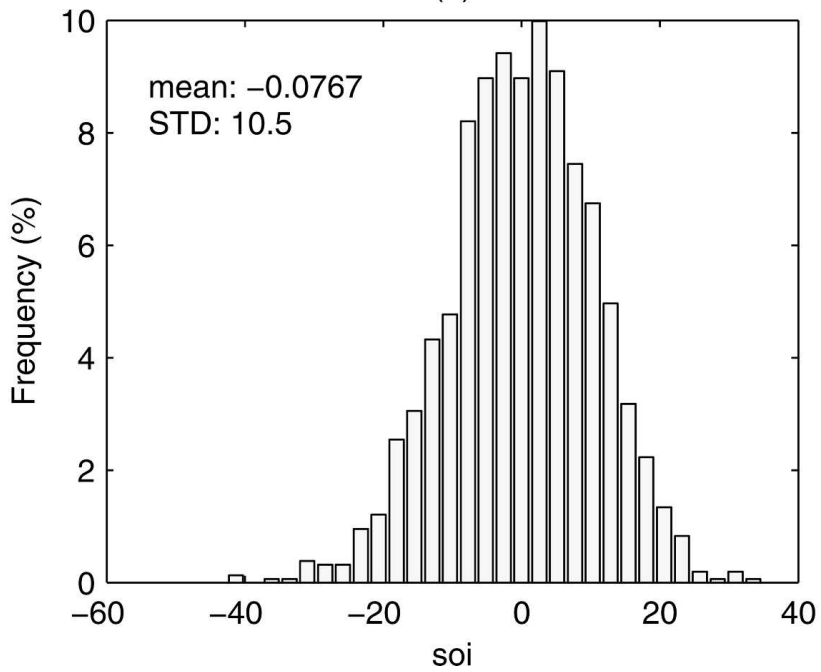

FIG. 2. Histograms of the climate indices for the (a) AO, (b) AAO, (c) NAO, (d) PNA, and (e) SO. 
a given increment $\rho$. To do so, the FPT concept is used. As the index data run through the past history, the accumulated values of the FPTs form the PDF (called FPT density function). The mode of the PDF shows the most probable time when the index decreases with the value of $\rho$ at the first time.

The FPT is important from a prediction point of view in several ways. First, although the climate indices are related to certain circulation patterns such as the negative SOI values generally connected to El Niño events, one still does not know the exact time of the El Niño onset. Therefore, the best one can do, from a statistical point of view, is to make a prediction at a time that is probabilistically favorable. This optimal time, as we will see, is determined by the maximum of the FPT density function, that is, the optimal FPT. Second, but not least, the FPT density function will by itself give invaluable, nontrivial information about the stochasticity of the climate indices. Third, FPT effectively represents the ocean-atmospheric model predictability (Chu et al. 2002a,b).

\section{FPT}

In a series of papers, Chu et al. (2002a) introduced the FPT $(\tau)$ concept into the ocean-atmospheric model predictability with the model error (i.e., "some quantity") first exceeding a predetermined tolerance level (i.e., "predetermined criterion"); they found that the FPT density function satisfied the backward FokkerPlanck equation; and they obtained the analytical solution of the FPT density function for the nonlinear simplified low-order Lorenz atmospheric model (Nicolis 1992). Both linear and nonlinear perspectives of forecast errors are investigated analytically (Chu et al. $2002 b$ ). Furthermore, the FPT was used to evaluate the full physical nowcast/forecast ocean prediction system. For example, the FPT density function is asymmetric with a broader and longer tail in a higher value side, which indicates long-term predictability. The long-term (extremely long) predictability is not an "outlier" and shares the same statistical properties as the short-term predictions (Chu et al. 2002c). The FPT is also used to verify the model's ability to predict Lagrangian drifter trajectories (Chu et al. 2004) and the regional ocean model predictability of stochastic perturbations in initial conditions, open boundary conditions, and winds (Chu and Ivanov 2005; Ivanov and Chu 2007).

Using the same concept, the FPT is used to explore the statistical features of the time series of climate indices. The features change either positively or negatively at a given time (Fig. 1). Then, of course, it is interested in predicting the exact change at a point in time. However, this is not possible. Therefore, the best one can do, from a statistical point of view, is to predict the time that is probabilistically favorable for the given index change. This optimal time is determined by the maximum of the first passage time distribution, that is, the optimal FPT.

Given a fixed value of an index reduction $(\rho)$, the corresponding time span (positive) is estimated for when the index reduction

$$
\gamma_{\Delta t}(t)=s(t+\Delta t)-s(t)
$$

reaches the level $\rho$ for the first time,

$$
\tau_{\rho}(t)=\inf \left\{\Delta t>0 \mid \gamma_{\Delta t}(t) \leq-\rho\right\},
$$

which is called the FPT. It is noted that a similar definition can be used for the index enhancement. As the index data run through the past history, which is represented by (2), the FPT density function satisfies the backward Fokker-Planck equation (Chu et al. 2002a):

$$
\frac{\partial p}{\partial t}-\left[D^{(1)}(\rho, t)\right] \frac{\partial p}{\partial \rho}-\frac{1}{2} \eta^{(2)} D^{(2)}(\rho, t) \frac{\partial^{2} p}{\partial \rho^{2}}=0 .
$$

The cumulative distribution is introduced for the transition time being larger than $\tau_{\rho}$, such that

$$
P\left(\tau_{\rho}\right)=\int_{\tau_{\rho}}^{\infty} p(\tau) d \tau .
$$

All five climate indices show similar Gaussian-type distributions (Fig. 2). The Brownian fluctuations identified in the NAO monthly index (Collette and Ausloos 2004) may extend to the other indices. For the Brownian fluctuations with zero drift, the FPT density function has the analytical solution for finite $\rho$ (Rangarajan and Ding 2000),

$$
p(\tau)=\frac{1}{\sqrt{\pi}} \frac{a}{\tau^{3 / 2}} \exp \left(-\frac{a^{2}}{\tau}\right), \quad a>0, \quad \tau>0 .
$$

Here, $a$ is a function of $\rho$. With $\rho \rightarrow 0$ as a limit case (i.e., no index reduction), the FPT tends toward 0 with a probability of $100 \%$. Thus, the FPT density function cannot be represented by (7) but by the $\delta$ function,

$$
p(\tau) \rightarrow \delta(\tau), \quad \text { as } \quad \rho \rightarrow 0 .
$$

The maximum value of $p(\tau)$ (for finite $\rho$ ) indicates the most favorable FPT,

$$
\tau_{\rho}^{(\max )}=\frac{2 a^{2}}{3} .
$$

With the known FPT density function (7), the $k$ th moment $(k=1,2, \ldots)$ of FPT for finite $\rho$ is calculated by 
(a) ao: $\rho=1.9 \quad a=1.81$

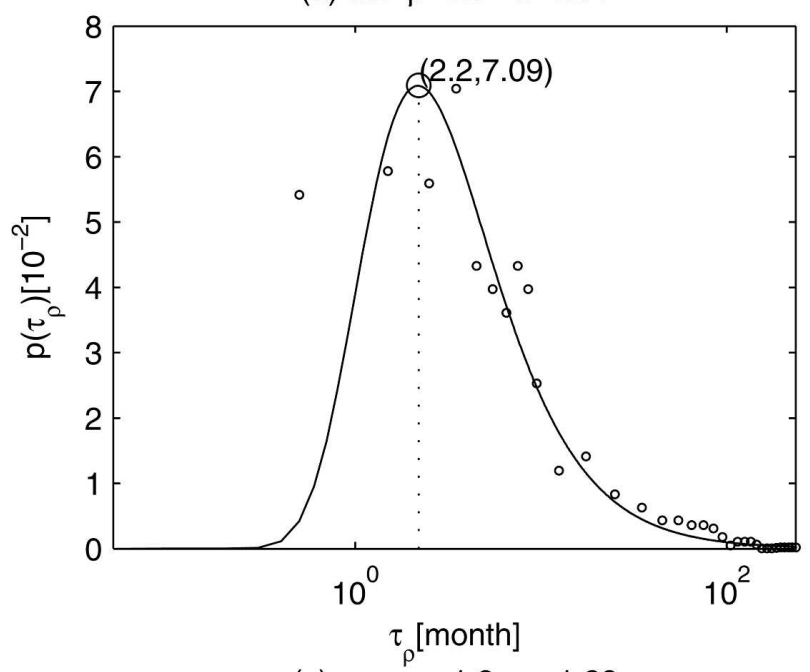

(c) nao: $\rho=1.9 \quad a=1.29$

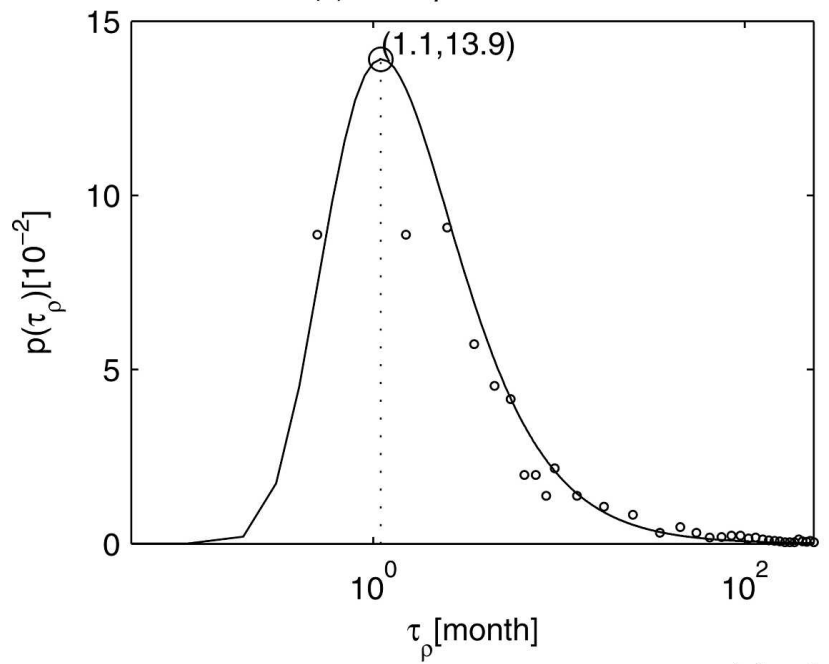

(b) aao: $\rho=1.9 \quad a=1.88$

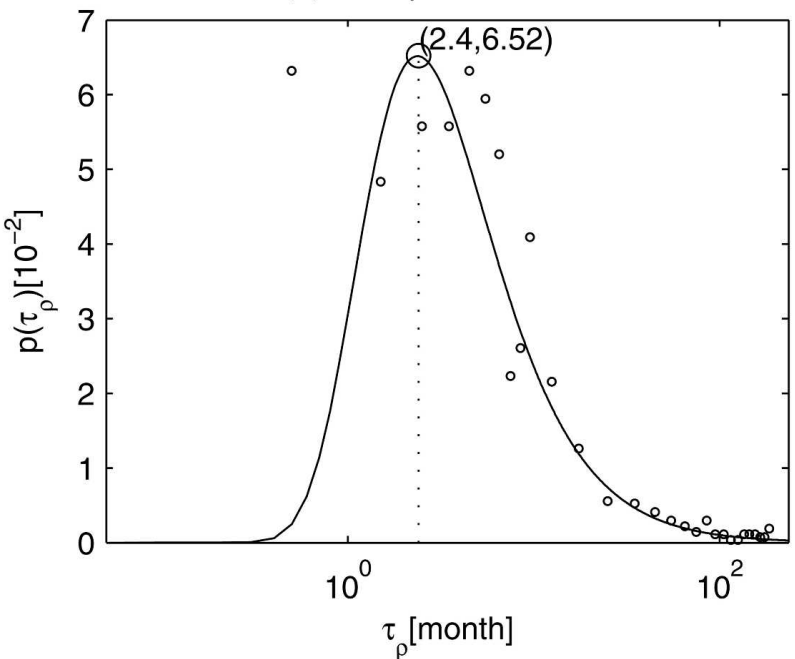

(d) npa: $\rho=1.9 \quad a=1.31$

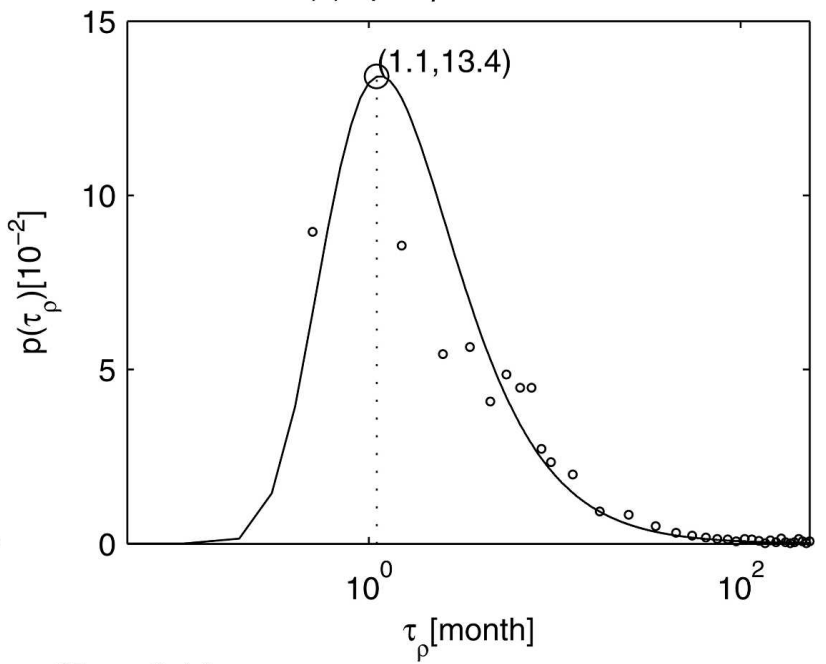

(e) soi: $\rho=25 \quad a=3.14$

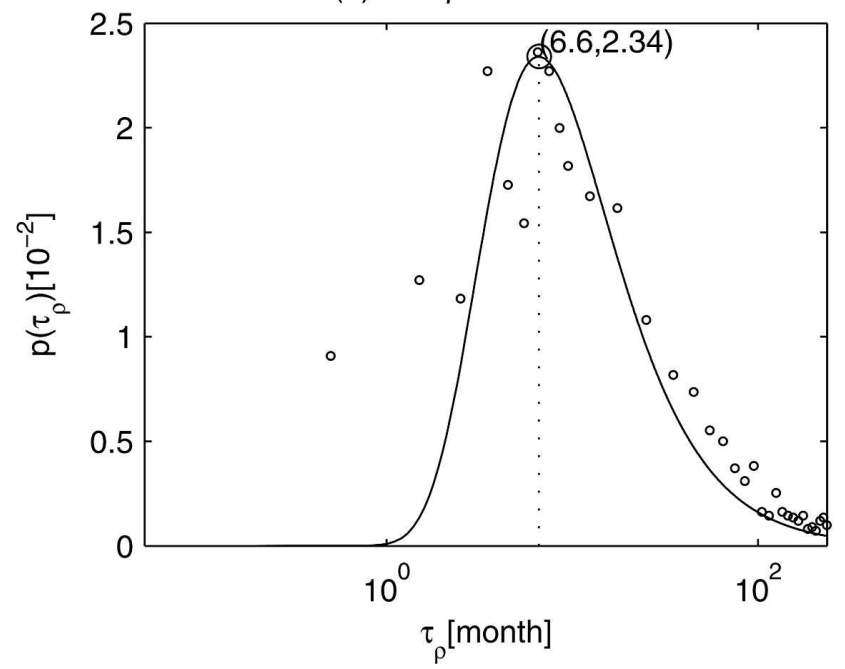

FIG. 3. FPT density functions with particular index reduction $\rho$ for the (a) AO, (b) AAO, (c) NAO, (d) PNA, and (e) SO. 
(a) ao: $a=0.43+0.695^{*} \rho$

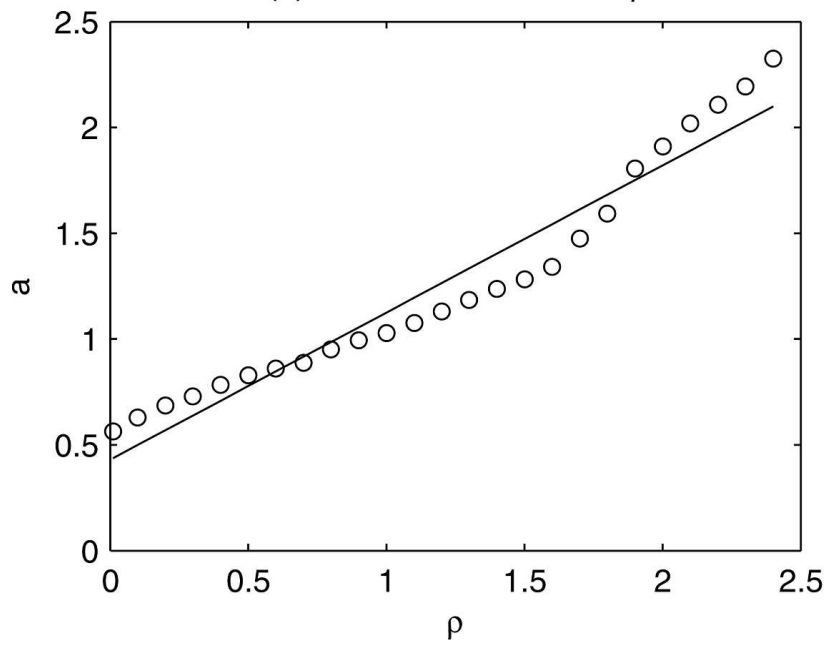

(c) nao: $\mathrm{a}=0.604+0.351^{*} \mathrm{\rho}$

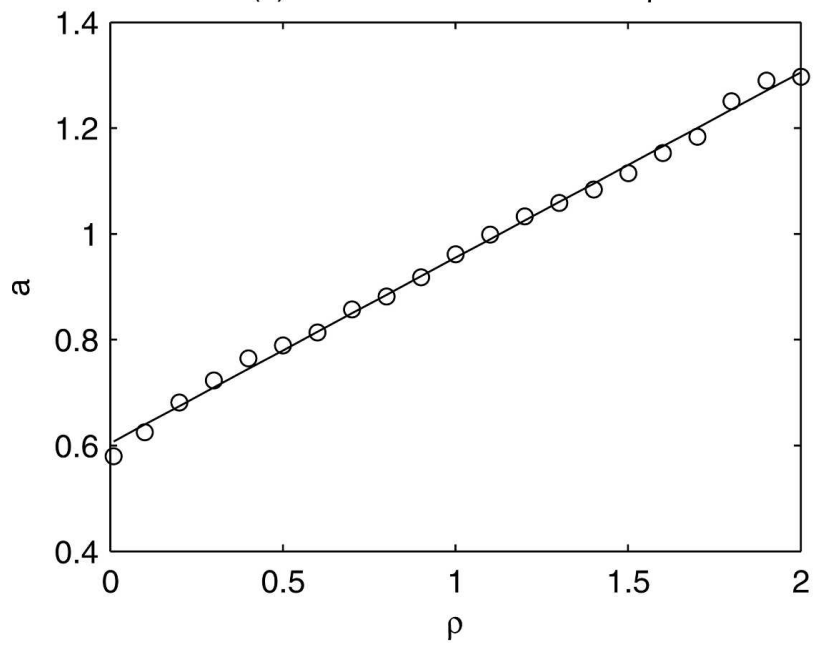

(b) aao: $a=0.508+0.562^{*} \rho$

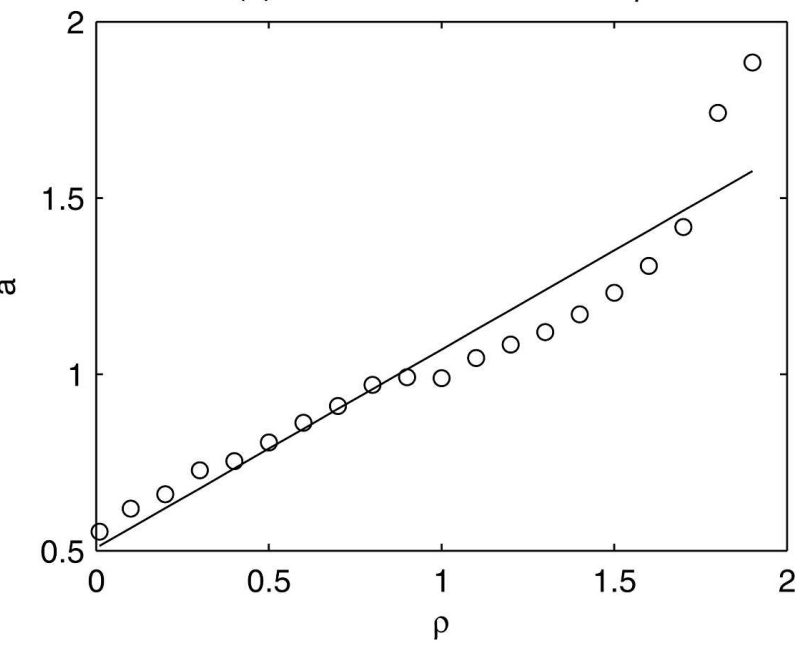

(d) npa: $a=0.6+0.373^{*} \rho$

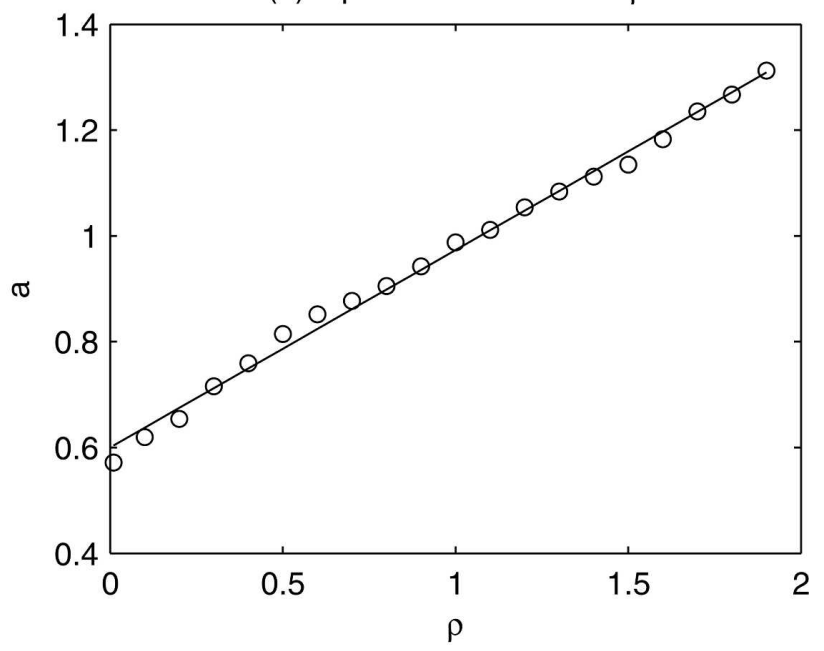

(e) soi: $a=0.165+0.125^{\star} \rho$

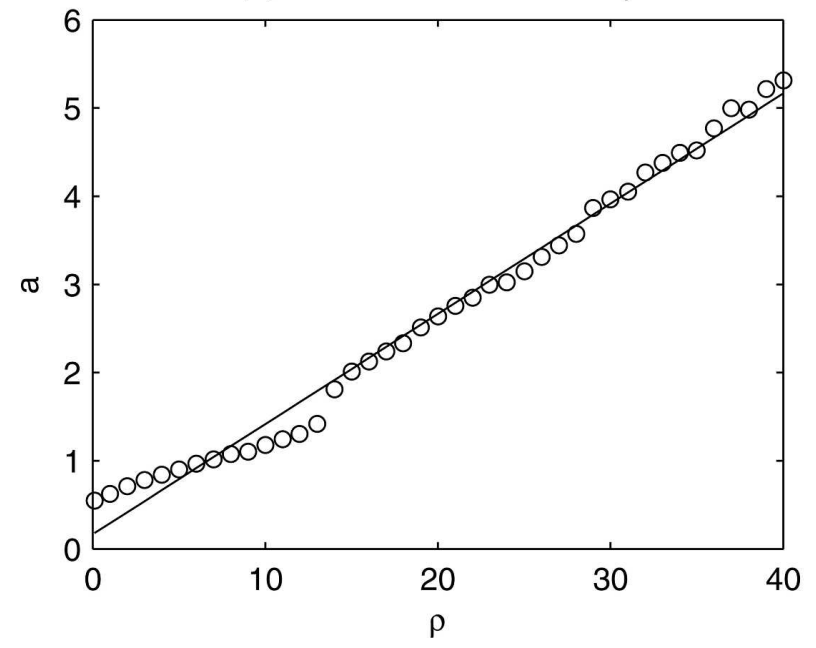

FIG. 4. Linear relationship between the parameter $a$ in the FPT density function and the index reduction $\delta$ for the (a) AO, (b) AAO, (c) NAO, (d) PNA, and (e) SO. 


$$
M_{k}(\rho)=k \int_{0}^{\infty} p(\tau) \tau^{k-1} d \tau, \quad k=1, \ldots, \infty .
$$

The mean and variance of FPT can be calculated from the first two moments:

$$
\begin{aligned}
\left\langle\tau_{\rho}\right\rangle & =M_{1}, \\
\left\langle\delta \tau_{\rho}^{2}\right\rangle & =M_{2}-M_{1}^{2},
\end{aligned}
$$

where the bracket denotes the average over realizations.

\section{Sensitivity of $p\left(\tau_{\rho}\right)$ with respect to $\rho$}

The theoretical distribution of (7) depends solely on the parameter $a$. This parameter can be determined from the monthly index data. First, the FPT density functions are constructed and fitted to (7) for each index with various values of index reduction $\rho$. For example, Fig. 3 shows $p\left(\tau_{\rho}\right)$ with $\rho=1.9$ for the AO, AAO, NAO, and PNA indices and with $\rho=25$ for the SOI. Note that almost an order of difference in $\rho$ values (1.9 versus 25$)$ is selected. This is due to the different ranges of index fluctuation: $(-4,4)$ for the $\mathrm{AO}, \mathrm{AAO}$, NAO, and PNA indices, and $(-40,40)$ for the SOI. It exhibits a rather well-defined and pronounced maximum, followed by an extended tail for very long FPTs indicating a nonzero and important probability of large passage times (note that the $\tau_{\rho}$ axis is logarithmic). These long (toward El Niño for the SOI) FPTs reflect periods where the tropical Pacific is in a strong El Niño phase and needs a long period of time before finally coming to an even stronger El Niño. The short FPTs on the other hand - those around the maximum-are in La Niña periods, which appears to be the most common scenario.

Second, for each index reduction $(\rho)$, parameter $(a)$ is determined from the $\tau_{\rho}$ data fitted to (7). From the scatter diagram for each index (Fig. 4), the linear relationship between $a$ and $\rho$,

$$
a=\alpha_{1}+\alpha_{2} \rho,
$$

is found using the least squares method. Here, Eq. (12) represents the NAO, NPA, and SOI indices quite well, but not so well for the AO and AAO indices because oscillations are found around the linear trend. Despite oscillations, the linear correlation between $a$ and $\rho$ is very evident with the positive regression coefficient $\alpha_{2}$ (Table 1).

To better understand the tail of this distribution, various values of $\rho$ are considered. If this level is small enough, it is likely that the index reduction will break through the level after the first month while larger
TABLE 1. Coefficients $\left(\alpha_{1}, \alpha_{2}\right)$ in Eq. (12) for the five climate indices.

\begin{tabular}{lccccc}
\hline & AO & AAO & NAO & PNA & SO \\
\hline$\alpha_{1}$ & 0.430 & 0.508 & 0.604 & 0.600 & 0.165 \\
$\alpha_{2}$ & 0.696 & 0.562 & 0.351 & 0.373 & 0.125 \\
\hline
\end{tabular}

FPTs will become more and more unlikely. However, the probability for a large FPT value will not be zero; if, say, it is a small-level $\rho$, then a period of strong El Niño will result in a $\tau_{\rho}(t)$ that might be considerably longer than one month since it takes time to recover from the El Niño event. For instance, after the 1992 El Niño event, it took five years to reach a new El Niño event in 1997.

\section{Characteristics of the index variability- Brownian motion}

A random process is called fractional Brownian motion if its cumulative FPT density function satisfies the power law (Ding and Yang 1995)

$$
P(\tau) \sim \tau^{H-1},
$$

with $0<H<1$. Here $H$ is the Hurst exponent. For $H=1 / 2$, the random process is the ordinary Brownian motion. The cumulative distribution, $P\left(\tau_{\rho}\right)$, for climate index data (Fig. 5) shows a power-law feature in the tail of the distribution scales. For a very small value of the index reduction, $\rho_{0}(0.01$ for the $\mathrm{AO}, \mathrm{AAO}, \mathrm{NAO}$, and PNA indices and 0.1 for the SOI), the cumulative FPT density function calculated from the index data shows that

$$
P\left(\tau_{0}\right) \sim \tau_{0}^{-\alpha_{0}}
$$

with $\alpha_{0} \sim 1 / 2$. Since the Hurst exponent of an ordinary Brownian motion is $H=1 / 2$, the empirically observed scaling (see Fig. 5) is a consequence of (at least close to) the Brownian motion behavior of the climate indices. This argument of an unbiased Brownian motion is also strengthened by observing that (see Fig. 5)

$$
P\left(\tau_{0}=1\right) \sim 1 / 2 .
$$

This indicates that the climate index has a $50 \%$ chance of increase or decrease at each time step (one month).

Figure 5 also shows the cumulative distributions $P\left(\tau_{\rho}\right)$ for different values of $\rho$ : for the AO, AAO, NAO, and PNA indices, $\rho=0.5,1.0$, and 1.5 ; for the SOI, $\rho=$ 10,20 , and 30 . From this figure it is seen that the tail exponent $\alpha_{\rho}$ is rather insensitive to the value of $\rho$. In 
(a): ao

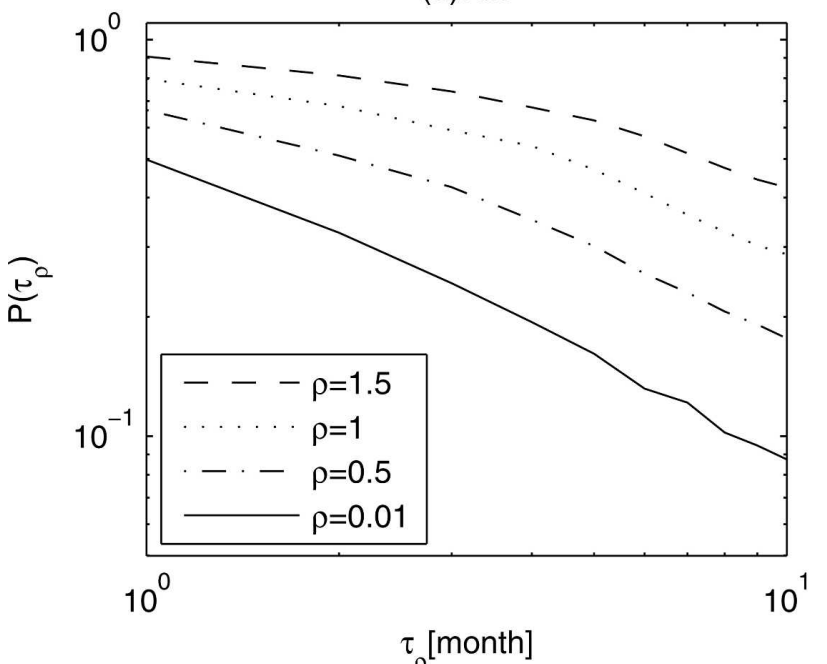

(c): nao

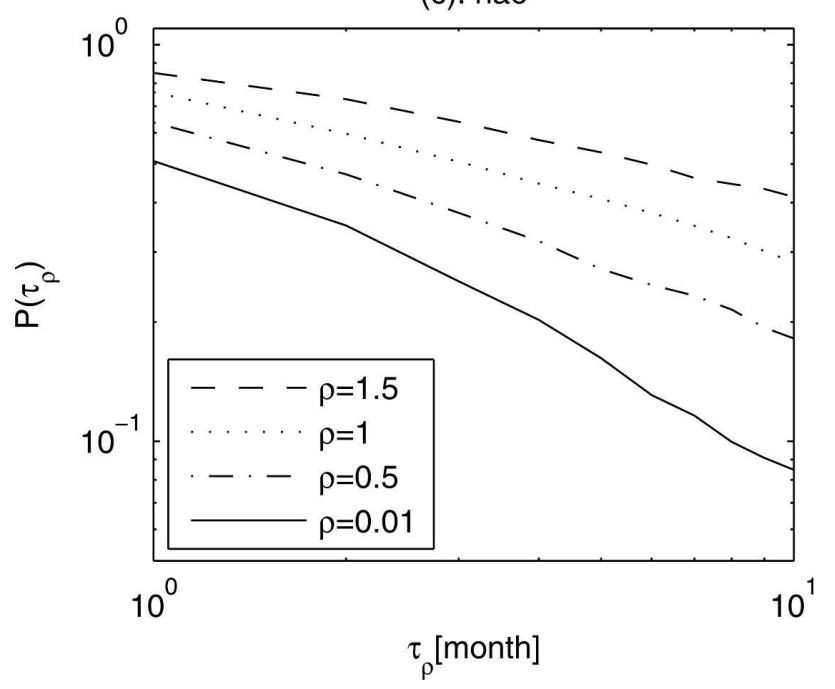

(b): aao

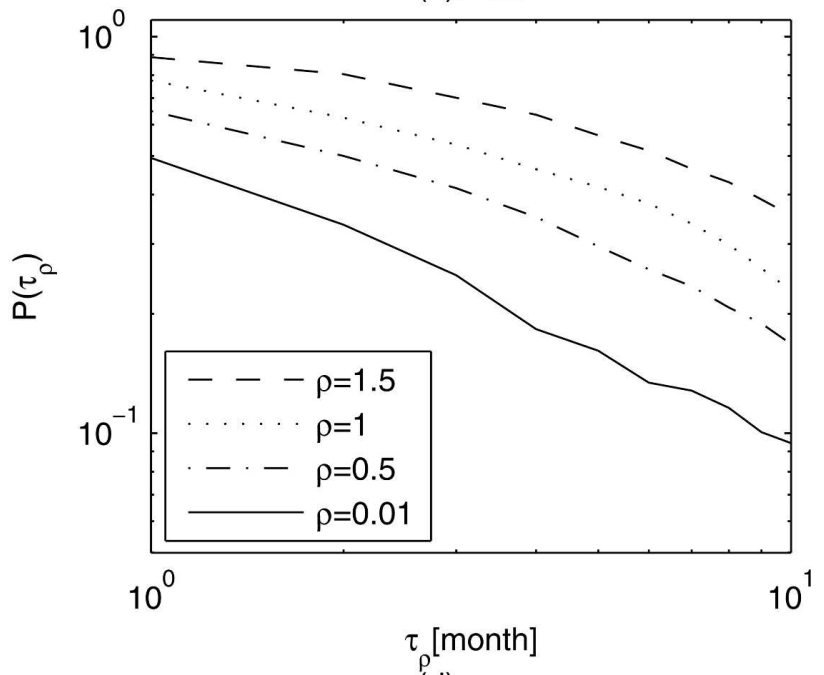

(d): pna

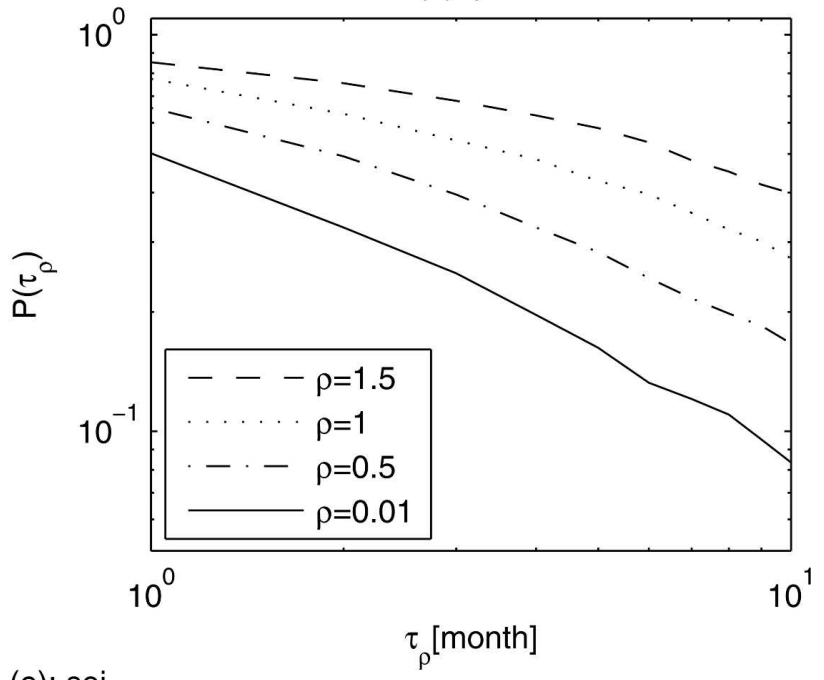

(e): soi

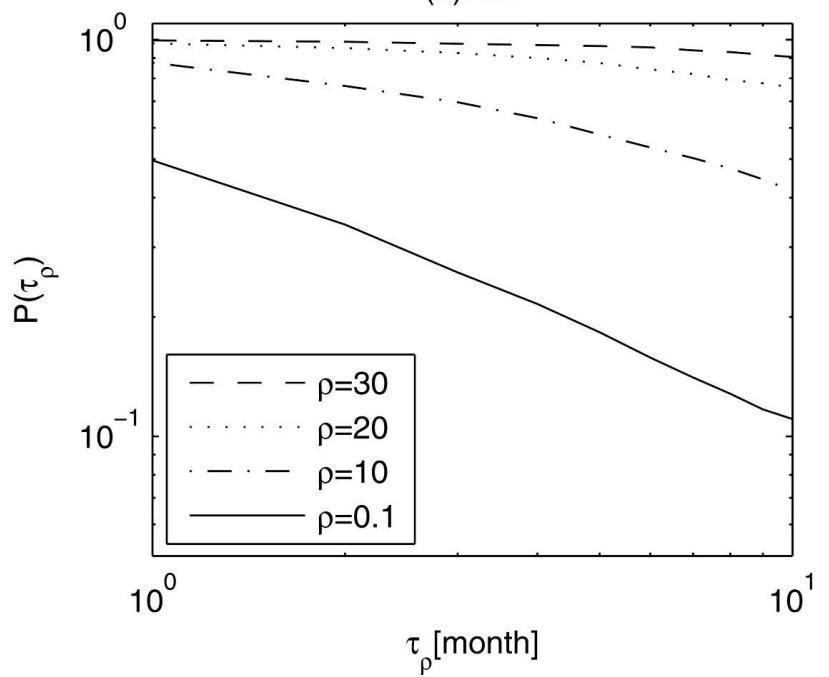

FIG. 5. The empirical cumulative density function $P\left(\tau_{\rho}\right)$ for different values of the index reduction $\rho$ for the (a) AO, (b) AAO, (c) NAO, (d) PNA, and (e) SO. It shows the power-law features. 
(a) soi: $\rho=15 \quad a=2.01$

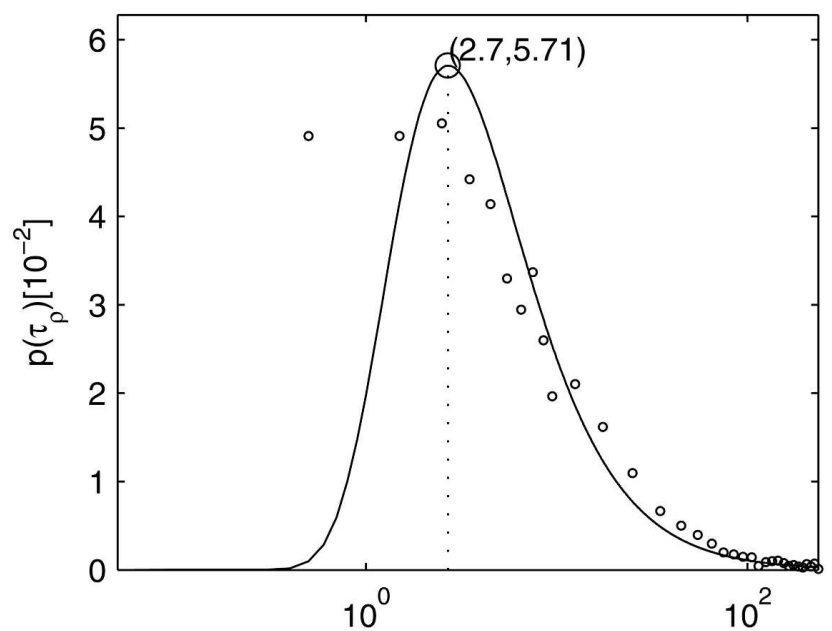

(c) soi: $\rho=25 \quad a=3.14$

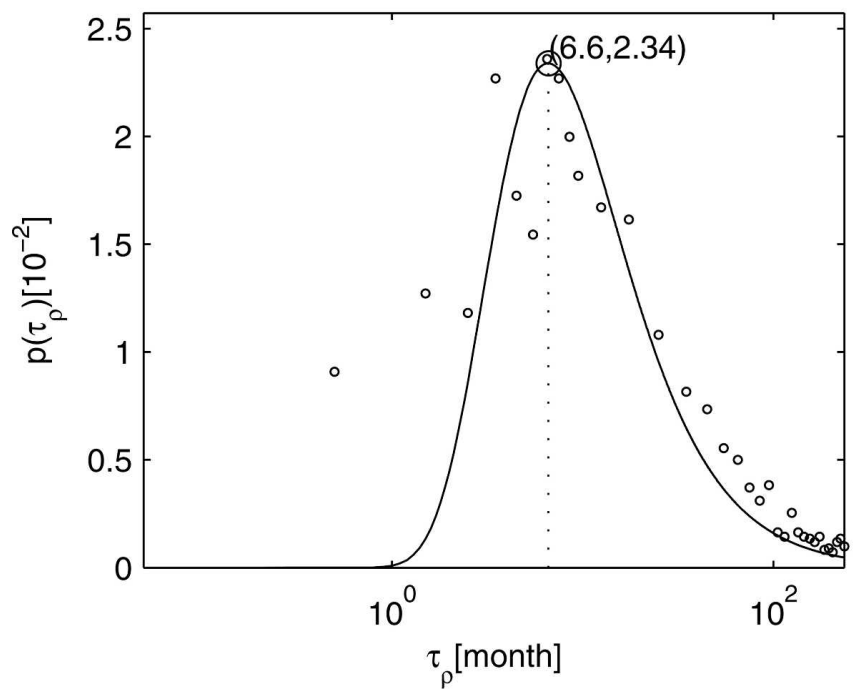

(b) soi: $\rho=20 \quad a=2.64$

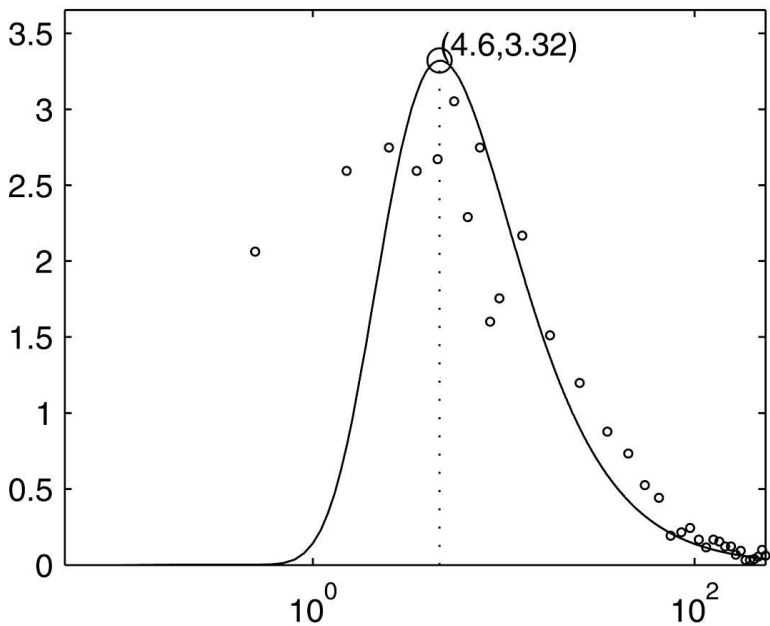

(d) soi: $\rho=30 \quad a=3.96$

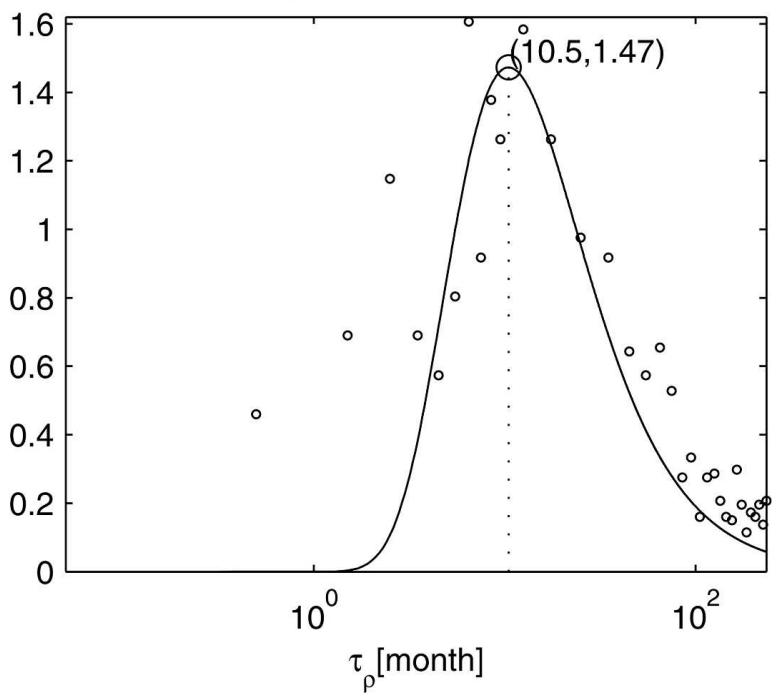

FIG. 6. FPT density function of the SOI with different values of $\rho$ : (a) 15 , (b) 20, (c) 25, and (d) 30. It is noted that the optimal FPT increases as $\rho$ increases.

particular one finds that $\alpha_{\rho} \sim 1 / 2$ over a broad range of values for $\rho$, which is consistent with the Brownian motion hypothesis.

Moreover, it is noted that as the level of $\rho$ is increased from zero, the optimal FPT $\left[\tau_{\rho}^{(\max )}\right]$ moves away from $\tau_{0}$ $=1$ and toward larger values. How does the optimal FPT $\tau_{\rho}^{(\max )}$ depend on the level of $\rho$ for a large $\rho$ ? This dependence, as measured from the empirical distribution (i.e., the histogram of FPT), is shown in Fig. 6. Intuitively, it is clear that the optimal FPT will increase as $\rho$ increases.

The power law is found for the optimal FPT versus $\rho$,

$$
\tau_{\rho}^{(\max )} \sim \rho^{\gamma} \quad \text { for } \quad \text { large } \rho
$$

with $\gamma \sim 2.0$ for the $\mathrm{AO}, \mathrm{AAO}$, and $\mathrm{SO}$ indices and $\gamma \sim 1.0$ for the NAO and PNA indices (see Fig. 7), which is consistent with the results for a Brownian motion with the theoretical FPT density function [see (7) and (8)]. Substituting (12) into (8) gives

$$
\tau_{\rho}^{(\max )}=\alpha_{2}^{2} \rho^{2}+2 \alpha_{1} \alpha_{2} \rho+\alpha_{1}^{2} .
$$

The exponent of the power law depends on the regression coefficients $\alpha_{1}$ and $\alpha_{2}$ (see Table 1 ). A large $\rho$ value usually means much larger than 1 for the SOI, and slightly larger than 1 for the AO, AAO, NAO, and PNA indices. For the SOI, the regression coefficients are given by $\alpha_{1}=0.165$ and $\alpha_{2}=0.125$, the leading term of (17) is $\alpha_{2}^{2} \rho^{2}$, and therefore the exponent of the 
(a): ao

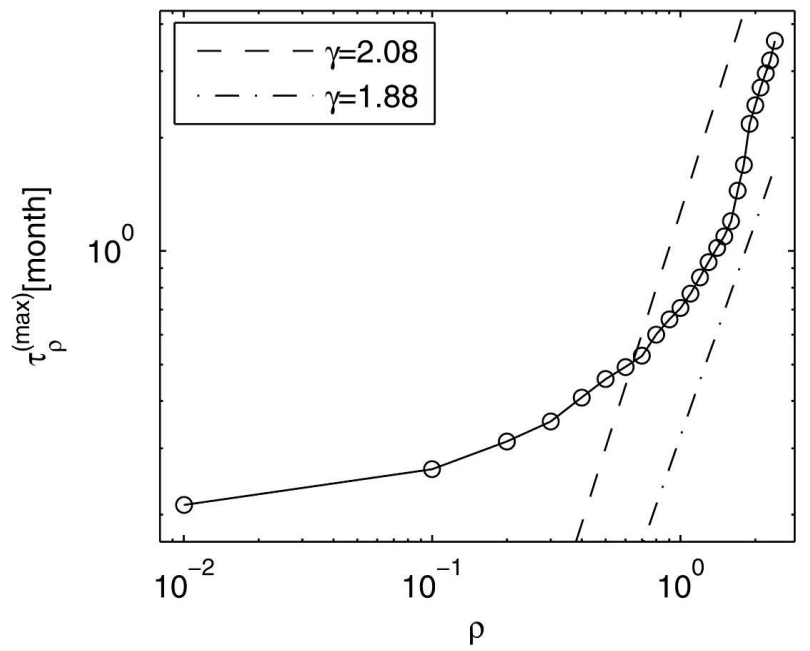

(c): nao

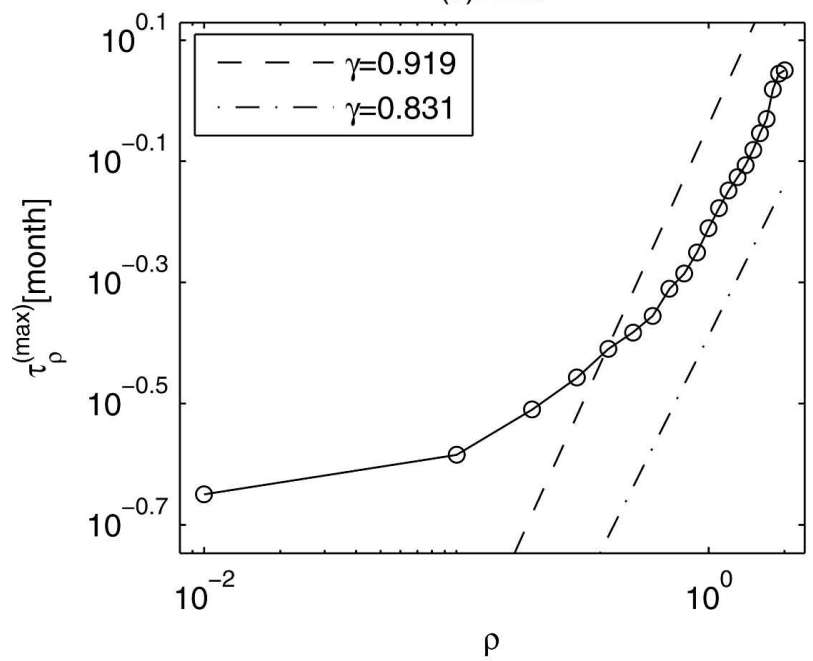

(b): aao

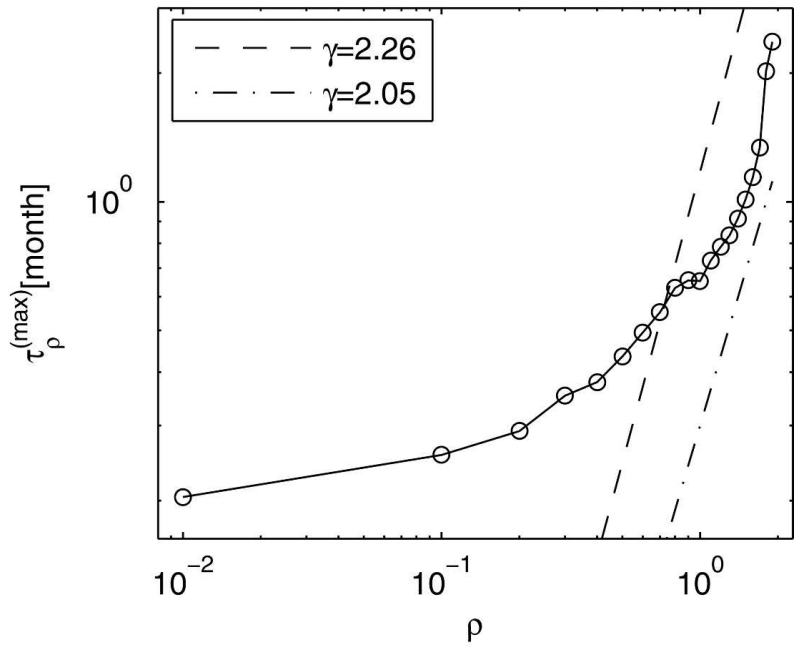

(d): pna

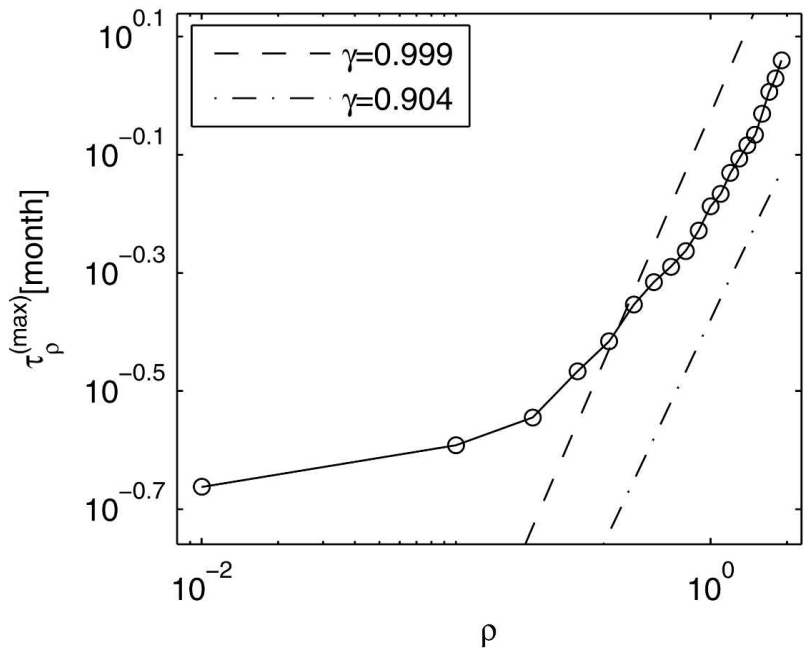

(e): soi

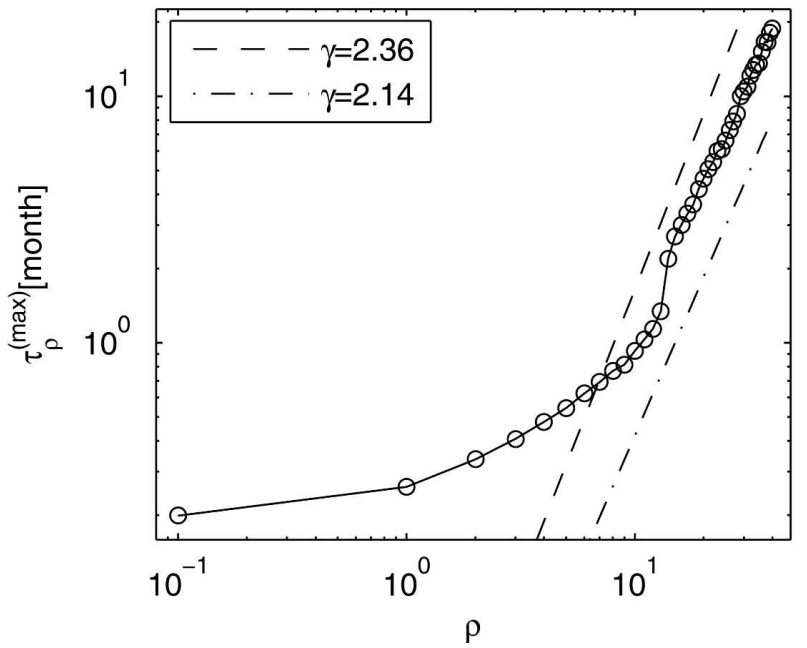

FIG. 7. Dependence of the optimal FPT $\tau_{\rho}^{(\max )}$ on the index reduction $\rho$ for the (a) AO, (b) AAO, (c) NAO, (d) PNA, and (e) SO. Power law exists for large $\rho$ values. 
power law is around 2. For the $\mathrm{AO}$ and $\mathrm{AAO}$ indices, $\alpha_{2}$ is larger than $\alpha_{1}$, the leading term of (17) is $\alpha_{2}^{2} \rho^{2}$ for a large $\rho$ value, and therefore the exponent of the power law is around 2. However, for the NAO and PNA indices, $\alpha_{2}$ is smaller than $\alpha_{1}$, the leading term of (17) is $2 \alpha_{1} \alpha_{2} \rho$ for a large $\rho$ value, and therefore the exponent of the power law is around 1. Not surprisingly, there are some deviations from standard theories for the variation of the optimal FPT with the level $\rho$ (i.e., the straight line). Furthermore, we have checked and found that there is an approximate symmetry under

$$
\rho \rightarrow-\rho
$$

for the FPT density function. One therefore does not have to consider index enhancement explicitly.

\section{Conclusions}

1) FPT presents a new way to detect the temporal variability of the climate indices. It predicts a typical time span $(\tau)$ needed to generate an index reduction of a given increment $\rho$. FPT is a random variable whose density function satisfies the backward Fokker-Planck equation. Solving this equation, it is easy to obtain the ensemble mean and variance of the FPT of the climate indices.

2) FPTs for the five climate indices show the Brownian fluctuations. This means that the early results of the Brownian fluctuations for the NAO index (e.g., Collette and Ausloos 2004) are also valid for the other indices ( $\mathrm{AO}, \mathrm{AAO}, \mathrm{PNA}$, and $\mathrm{SO}$ ). With $\delta \rightarrow 0$ as a limit case (i.e., no index reduction), the FPT density function tends to the $\delta$ function.

3) For a very small value of the index reduction, $\rho_{0}$ ( 0.01 for the AO, AAO, NAO, and PNA indices and 0.1 for the SOI), the cumulative FPT density function, shows the power-law dependence on $\tau_{\rho}$ with the exponent approximately $-1 / 2$. Another wellknown method to check power-law dependence is examination of the autocorrelation function. This also confirms that the climate indices have the Brownian-type fluctuations.

4) The optimal FPT has a power-law dependence on the index reduction $(\rho)$ for large-value $\rho$ with the exponent $(\sim 2)$ for the $\mathrm{AO}, \mathrm{AAO}$, and $\mathrm{SO}$ indices and $(\sim 1)$ for the NAO and PNA indices.

5) The FPT density functions as well as the variation of the optimal FPT can be applied if one wants to estimate the most probable time period needed for the low-frequency atmospheric circulation pattern to be sustained if a prediction aims at a specific optimal transition.
6) The FPT analysis can be applied to other air-ocean time series.

Acknowledgments. Chenwu Fan is highly appreciated for invaluable comments and computational assistance. This work was supported by the Office of Naval Research and the Naval Postgraduate School.

\section{REFERENCES}

Ausloos, M., and K. Ivanova, 2001: Power-law correlations in the southern-oscillation-index fluctuations characterizing El Niño. Phys. Rev. E, 63, 047201, doi:10.1103/PhysRevE. 63.047201 .

Barnston, G., and R. E. Livezey, 1987: Classification, seasonality and low-frequency atmospheric circulation patterns. Mon. Wea. Rev., 115, 1083-1126.

Chu, P. C., and L. M. Ivanov, 2005: Statistical characteristics of irreversable predictability time in regional ocean models. Nonlinear Processes Geophys., 12, 1-10.

$\longrightarrow,-$, and C. W. Fan, 2002a: Backward Fokker-Planck equation for determining model valid prediction period. J. Geophys. Res., 107, 3058, doi:10.1029/2001JC000879.

_ — - T. M. Margolina, and O. V. Melnichenko, 2002b: On probabilistic stability of an atmospheric model to various amplitude perturbations. J. Atmos. Sci., 59, 2860-2873.

- - - - L. H. Kantha, O. V. Melnichenko, and Y. A. Poberezhny, 2002c: Power law decay in model predictability skill. Geophys. Res. Lett., 29, 1748, doi:10.1029/ 2002GL014891.

,,,--- T. M. Margolina, O. V. Melnichenko, and Y. A. Poberenzhny, 2004: Lagrangian predictabilty of highresolution regional ocean models. Nonlinear Processes Geophys., 11, 47-66.

Collette, C., and M. Ausloos, 2004: Scaling analysis and evolution equation of the North Atlantic oscillation index fluctuations. Int. J. Mod. Phys. C, 15, 1353-1366.

Ding, M., and W. Yang, 1995: Distribution of the first return time in fractional Brownian motion and its application to the study of on-off intermittency. Phys. Rev. E, 52, 207-213.

Drosdowsky, W., 1994: Analog (nonlinear) forecasts of the Southern Oscillation index time series. Wea. Forcasting, 9, 78-84.

Greatbatch, R. J., 2000: The North Atlantic Oscillation. Stochastic Environ. Res. Risk Assess., 14, 213-242.

Ivanov, L. M., and P. C. Chu, 2007: On stochastic stability of regional ocean models to finite-amplitude pertubations of initial conditions. Dyn. Atmos. Oceans, 43, 199-225.

Keppenne, C. L., and M. Ghil, 1992: Adaptive spectral analysis and prediction of the Southern Oscillation index. J. Geophys. Res., 97, 20 449-20 454.

Lind, P. G., A. Mora, J. A. C. Gallas, and M. Haase, 2005: Reducing stochasticity in the North Atlantic Oscillation index with coupled Langevin equations. Phys. Rev. E, 72, 056706, doi:10.1103/PhysRevE.72.056706.

Maharaj, E. A., and M. J. Wheeler, 2005: Forecasting an index of the Madden-Julian Oscillation. Int. J. Climatol., 25, 16111618.

Nicolis, C., 1992: Probabilistic aspects of error growth in atmospheric dynamics. Quart. J. Roy. Meteor. Soc., 118, 553-568.

Palmer, T. N., 2000: Predicting uncertainty in forecasts of weather and climate. Rep. Prog. Phys., 63, 71-116. 
Rangarajan, G., and M. Ding, 2000: First passage time distribution for anomalous diffusion. Phys. Lett. A, 273, 322-330.

Rimmington, G. M., and N. Nicholls, 1993: Forecasting wheat yields in Australia with the Southern Oscillation index. Aust. J. Agric. Res., 44, 625-632.

Ropelewski, C. F., and M. S. Halpert, 1987: Global and regional scale precipitation patterns associated with the El Niño/ Southern Oscillation. Mon. Wea. Rev., 115, 1606-1626.

Smith, S. R., and C. R. Sterns, 1993: Antarctic pressure and temperature anomalies surrounding the minimum in the Southern Oscillation index. J. Geophys. Res., 98, 61-78.
Torrence, C., and G. P. Campo, 1998: A practical guide to wavelet analysis. Bull. Amer. Meteor. Soc., 79, 62-78.

Walker, G. T., and E. W. Bliss, 1937: World weather VI. Mem. Roy. Meteor. Soc., 4, 119-139.

Wallace, J. M., 2000: North Atlantic oscillation/annular mode: Two paradigms-One phenomenon. Quart. J. Roy. Meteor. Soc., 126, 791-805.

Wanner, H., S. Bronnimann, C. Casty, D. Gyalistras, J. Luterbacher, C. Schmutz, D. B. Stephenson, and E. Xoplaki, 2001: North Atlantic oscillation-Concepts and studies. Surv. Geophys., 22, 321-382. 